\title{
Impulsive radio and hard X-ray emission from an M-class flare
}

\author{
Ping Zhang ${ }^{1,2,4}$, Yang $\mathrm{Guo}^{3}$, Lu Wang ${ }^{1,2}$, and Siming Liu ${ }^{1}$ \\ 1 Key Laboratory of Dark Matter and Space Astronomy, Purple Mountain Observatory, Chinese Academy of Sciences, \\ Nanjing 210008, PR China \\ e-mail: liusm@pmo.ac.cn, zhangping@pmo.ac.cn \\ 2 University of Chinese Academy of Sciences, Yuquan Road 19, Beijing 100049, PR China \\ 3 School of Astronomy and Space Science, Nanjing University, Nanjing 210023, PR China \\ ${ }^{4}$ Paul Scherrer Institut, Villigen PSI 5232, Switzerland \\ e-mail: ping. zhang@psi.ch
}

Received 30 May 2017 / Accepted 25 January 2018

\begin{abstract}
Context. Impulsive radio and hard X-ray emission from large solar flares are usually attributed to a hard distribution of high-energy electrons accelerated in the energy dissipation process of magnetic reconnection.

Aims. We report the detection of impulsive radio and hard X-ray emissions produced by a population of energetic electrons with a very soft distribution in an M-class flare: SOL2015-08-27T05:45.

Methods. The absence of impulsive emission at $34 \mathrm{GHz}$ and hard X-ray emission above $50 \mathrm{keV}$ and the presence of distinct impulsive emission at $17 \mathrm{GHz}$ and lower frequencies and in the $25-50 \mathrm{keV}$ X-ray band imply a very soft distribution of energetic electrons producing the impulsive radio emission via the gyro-synchrotron process, and impulsive X-rays via bremsstrahlung.

Results. The spectrum of the impulsive hard X-ray emission can be fitted equally well with a power-law model with an index of $\sim 6.5$ or a super-hot thermal model with a temperature as high as $100 \mathrm{MK}$. Imaging observations in the extreme-UV and X-ray bands and extrapolation of the magnetic field structure using a nonlinear force-free model show that energetic electrons trapped in coronal loops are responsible for these impulsive emissions.

Conclusions. Since the index of the power-law model is nearly constant during the impulsive phase, the power-law distribution or the super-hot component should be produced by a bulk energization process such as the Fermi and betatron acceleration of collapsing magnetic loops.
\end{abstract}

Key words. acceleration of particles - plasmas - radiation mechanisms: thermal

\section{Introduction}

Solar flares are triggered by magnetic reconnection on small kinetic scales, where magneto-hydrodynamic (MHD) description of magnetized plasmas is not appropriate. However, such smallscale processes can lead to change in the topology of largescale magnetic field structures whose evolution can be accurately modelled with MHD and may dominate the release of magnetic energy stored in nonpotential fields (Musset et al. 2015). It has been recognized that the released magnetic energy can be converted into the kinetic energy of high-energy particles and/or hot plasmas via a variety of processes that are closely coupled to the magnetic field structure (Somov \& Kosugi 1997) and can be probed via observations of radiative characteristics of these highenergy particles. Many processes proposed for energization of the background plasma assume a particular magnetic field configuration and explore a certain parameter space of the magnetic energy release process. It is therefore essential to have a comprehensive observational coverage of individual flares to determine which process plays the dominant role in order to advance our understanding of solar flares (Somov et al. 2005; Liu et al. 2013; Sharykin et al. 2014).

Plasma energization by collapsing magnetic traps is one of the leading models proposed for solar flares and has been extensively explored (e.g., Somov \& Kosugi 1997;
Eradat Oskoui etal. 2014). The model predicts bulk energization of injected particles and an invariance of the power-law index for a power-law distribution of injected particles. If the injected particles have a thermal distribution, the trapped particle distribution will be close to a Maxwellian if the betatron [23.5pc] process associated with transverse contraction of magnetic loops dominates, and will be close to a power law with a high-energy cutoff, if the Fermi process associated with longitudinal contraction of magnetic loops dominates (Bogachev \& Somov 2007). Since the adiabatic approximation is assumed, the injected particles can be energized at most by the factor of the transverse contraction (Bogachev \& Somov 2005). It therefore has difficulties in accelerating particles to very high energies.

Impulsive hard X-ray emission from large solar flares (greater than GOES class M) usually originates from footpoints of flaring loops and is attributed to a hard nonthermal population of energetic electrons (Guo et al. 2011; Kim et al. 2012). However, if the plasma energization is dominated by collapsing magnetic traps, hard X-ray emission produced by high-energy electrons injected into footpoints via the thick-target model may be much weaker than emission produced by energetic electrons trapped in the flare loop via the thin-target model (Brown 1971; Bogachev \& Somov 2005) and the high-energy emission can be dominated by a soft distribution of energetic electrons trapped in the corona (Bogachev \& Somov 2007). 
We carry out detailed analyses of multiwavelength observations of an M-class flare that occurred on August 27, 2015. Like other large flares this flare has distinct impulsive and gradual emission components; however, the nondetection of impulsive radio emission at $34 \mathrm{GHz}$ and hard $\mathrm{X}$-ray emission above $50 \mathrm{keV}$ implies that the electron acceleration is not very efficient in the sense that very few electrons are accelerated to very high energies. Radio and X-ray spectral analyses show that the impulsive emission can be produced by high-energy electrons with either a very soft power-law distribution or a super-hot thermal distribution. Imaging observations at radio, X-ray, and extreme-UV (EUV) bands reveal a coronal source of the impulsive emission observed in radio and X-rays. The coronal origin of the impulsive emission is further confirmed via nonlinear force-free extrapolation of the magnetic field structure. The flare therefore may represent a case in which the plasma energization process is dominated by collapsing magnetic traps.

In Sect. 2 we present an observational overview of the light curves in the radio, EUV, and X-ray bands. In combination with imaging observations at $17 \mathrm{GHz}$ and $34 \mathrm{GHz}$ with the Nobeyama Radio Heliograph (NoRH), Sect. 3 is dedicated to separation of the gradual and impulsive radio emission components. Radio and X-ray spectral analyses are carried out in Sect. 4 to determine the nature of the impulsive emission. Extreme-UV and $\mathrm{X}$-ray imaging observations and magnetic field extrapolation are presented in Sect. 5 to confirm the association of impulsive emission with high-energy electrons trapped in flare loops. We discuss these results and draw conclusions in Sect. 6.

\section{Light curves}

The flare started near 4:40 UT on August 27, 2015, and has been observed by instruments on several satellites such as GOES, RHESSI, Hinode, Fermi, SDO, and IRIS. The GOES observations show that the 1-8 $\AA$ soft X-ray flux peaked near 05:45 UT more than one hour after the soft X-ray emission first started to increase (see the third panel of Fig. 1) with a peak value corresponding to a GOES class M2.9. The flare has an extended relatively stable pre-heating phase lasting from 4:40 UT to near 5:35 UT, when the main impulsive phase started. The fourth panel of Fig. 1 shows the temperature and emission measure derived from the GOES flux measurements with average fluxes from 4:30 UT to 4:32 UT selected as the backgrounds (Garcia 1994). In the pre-heating phase, the temperature varies between $10 \mathrm{MK}$ and $15 \mathrm{MK}$ and the emission measure increases very slowly. After the main impulsive phase, the temperature reaches a peak value of $18 \mathrm{MK}$ and the emission measure reaches a peak value of $\sim 1.0 \times 10^{49} \mathrm{~cm}^{-3}$. The emission measure increases by about one order of magnitude during the main impulsive phase from 5:35 UT to 5:45 UT.

From the emission measure and temperature, we can also obtain the bolometric flux of this hot plasma, which is indicated by the solid line in the third panel of Fig. 1, which also shows SDO/EVE measurement of flux variation in EUV bands. The bolometric flux is more than one order of magnitude higher than the flux in the 1-8 $\mathrm{\text {band}}$. The EUV fluxes are closely correlated with the soft X-ray fluxes measured by GOES and the 1-70 EVE fluxes are about one order of magnitude higher than the bolometric flux of the hot plasma inferred from GOES observations, indicating the presence of warm plasmas that produce very weak emission in the GOES energy bands (Chamberlin et al. 2012; Feng et al. 2013; Sharykin et al. 2014).

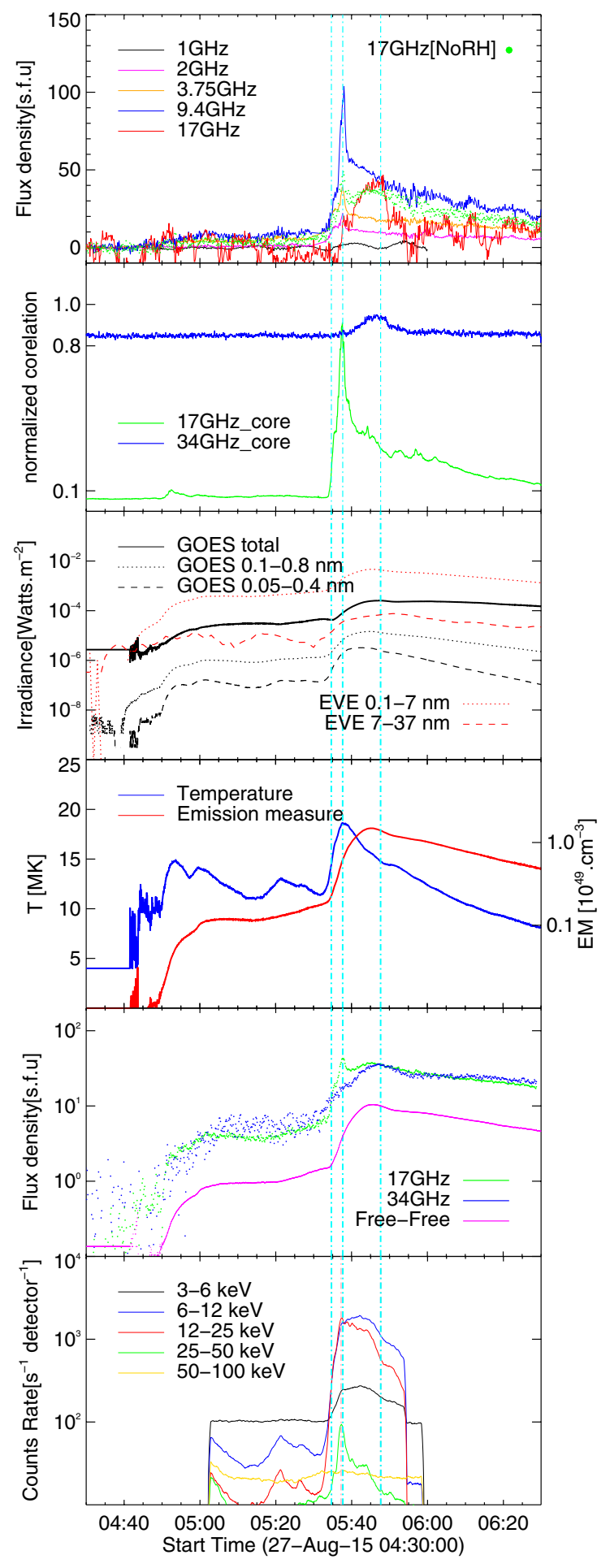

Fig. 1. Evolution of fluxes and model parameters for an M2.9 flare observed on August 27, 2005. From top to bottom: (1) radio light curves obtained with the NoRP and $17 \mathrm{GHz}$ light curve obtained with the NoRH; (2) normalized correlation coefficients of the NoRH observations at 17 and $34 \mathrm{GHz}$; (3) SDO/EVE light curves and GOES light curves, the solid line gives the bolometric flux of the isothermal plasma inferred from GOES flux measurements; (4) temperature and emission measure derived from GOES fluxes; (5) radio light curves at $17 \mathrm{GHz}$ and $34 \mathrm{GHz}$ obtained with the NoRH, the pink solid line gives the optically thin $17 \mathrm{GHz}$ radio flux density produced via the bremsstrahlung process by the isothermal plasma inferred from GOES observations; and (6) hard X-ray light curves obtained with the RHESSI. The vertical dot-dashed lines are drawn to facilitate the comparison between the panels. 
In the radio bands, the flare is well-covered by the Nobeyama Radio Heliograph (NoRH) and Polarimeter (NoRP). The first panel of Fig. 1 shows the light curves at 1, 2, 3.75, 9.4, and $17 \mathrm{GHz}$ obtained with the NoRP, and the $17 \mathrm{GHz}$ light curve obtained with the NoRH. The 4:30 UT-4:32 UT period is selected to obtain the background flux densities. The NoRH flux density is derived from radio images of the whole solar disk with the pre-flare background subtracted. To increase the signalto-noise ratio $(\mathrm{S} / \mathrm{N})$, NoRH radio observations are integrated for $10 \mathrm{~s}$ to obtain these images. As can be seen, the NoRH observations have a much higher $\mathrm{S} / \mathrm{N}$ than the NoRP flux density measurement at $17 \mathrm{GHz}$ mainly due to higher sensitivity of the NoRH.

Except at $1 \mathrm{GHz}$ where self-absorption is severe, the main phase of the flare is clearly detected, and significant flux increases can already be seen near 4:50 UT at several frequencies. Although the $17 \mathrm{GHz}$ flux density measurement with the NoRP shows weak evidence for an impulsive emission component due to low $\mathrm{S} / \mathrm{N}$, a distinct impulsive emission component is clearly seen in other bands and in NoRH imaging observations at $17 \mathrm{GHz}$. The flare was also observed by the Chinese solar radio spectrometer operating in the $12-16 \mathrm{GHz}$ band in Mengcheng, Anhui. Although the observations are consistent with the NoRP and NoRH measurements with the impulsive component detected, the quality of the flux density measurement is low due to the unstable electronic system of the instrument (Wang et al. 2017).

The second panel of Fig. 1 shows the correlation coefficients of NoRH observations normalized to the corresponding peak value. At $17 \mathrm{GHz}$, the correlation started to increase at 4:50 UT and reached its peak value at 5:37 UT. However, the correlation at $34 \mathrm{GHz}$ was very stable and started to increase only after 5:37 UT. There is no signature of an impulsive emission component at $34 \mathrm{GHz}$. The light curves of NoRH observations are shown in the fifth panel with a cadence of $10 \mathrm{~s}$ and an integration time of 2 min to suppress fluctuations of the flux density measurements of active region 12403 that produced this flare. While the impulsive component in the $17 \mathrm{GHz}$ light curve is evident, the $34 \mathrm{GHz}$ flux density evolves gradually without any signature associated with the impulsive emission, in agreement with the evolution of the correlation coefficients in the second panel.

It is interesting to note that the NoRH flux densities of the gradual emission component at $17 \mathrm{GHz}$ and $34 \mathrm{GHz}$ appear to be equal throughout the flare, which implies a bremsstrahlung (free-free) origin of this emission. The pink solid line in this panel shows the optically thin $17 \mathrm{GHz}$ free-free emission produced by the hot plasma inferred from GOES observations (Tsap et al. 2016). It shows gradual evolution and is about a factor of 3 lower than the radio flux density observed with the NoRH. This is consistent with the above-mentioned SDO/EVE observations (third panel), which reveal warm plasmas that can produce radio emission via the bremsstrahlung process but negligible emission in the GOES bands due to temperatures lower than those derived from GOES measurements (fourth panel).

The bottom panel of Fig. 1 shows corrected X-ray count rates obtained with RHESSI. The flare is evident below $50 \mathrm{keV}$. In addition a prominent impulsive component is seen in the $25-50 \mathrm{keV}$ light curve and is closely correlated with the impulsive component observed in the radio bands. Below $25 \mathrm{keV}$, the light curves show gradual evolution in agreement with the GOES, EVE, and $34 \mathrm{GHz} \mathrm{NoRH}$ observations.

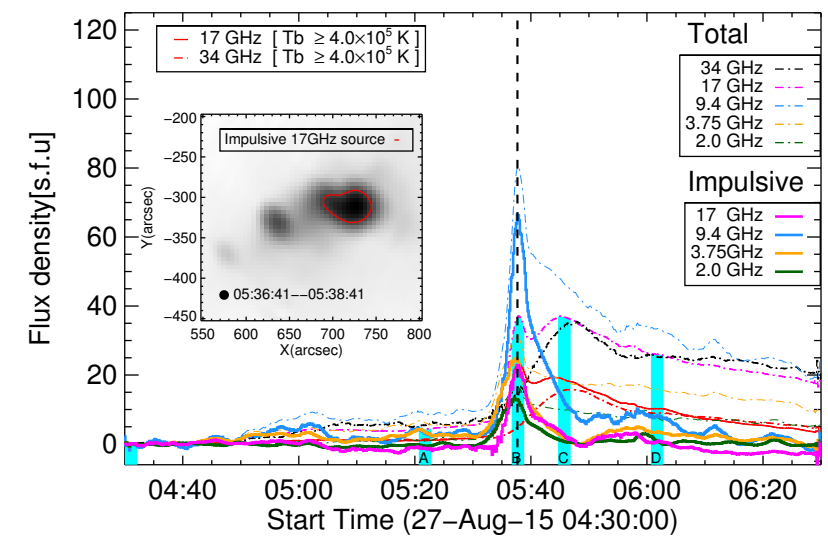

Fig. 2. Light curves of different emission components from NoRP $2 \mathrm{GHz}, 3.75 \mathrm{GHz}, 9.4 \mathrm{GHz}$, and NoRH $17 \mathrm{GHz}, 34 \mathrm{GHz}$. The dotdashed lines are for the 2-min averaged total emission and the solid lines are for the impulsive component. The thick pink and solid blue lines are obtained by subtracting the $34 \mathrm{GHz}$ flux density from the $17 \mathrm{GHz}$ and $9.4 \mathrm{GHz}$ flux densities, respectively. The red lines indicate emissions from a region with a brightness temperature higher than $4 \times 10^{5} \mathrm{~K}$ at the flux peak of the impulsive $17 \mathrm{GHz}$ emission. The corresponding image (see inset) is shown with the red contour indicating the brightness temperature of $4 \times 10^{5} \mathrm{~K}$. The cyan vertical bars mark the periods for the pre-flare background subtraction and for the four images shown in Fig. 3.

\section{Separation of the impulsive and gradual emission components}

To better understand the nature of the impulsive and gradual emissions and their relation, it is essential to separate these two emission components. The good correlation of the gradual emission component in different radio frequencies and the flat NoRH spectrum between $17 \mathrm{GHz}$ and $34 \mathrm{GHz}$ suggests an optically thin bremsstrahlung interpretation for the gradual component. The $34 \mathrm{GHz}$ NoRH light curve does not have an impulsive feature, unlike those at lower frequencies. We can obtain an estimate for the light curve of the impulsive component at $17 \mathrm{GHz}$ in NoRH and $9.4 \mathrm{GHz}$ in NoRP by subtracting the NoRH $34 \mathrm{GHz}$ flux density. Since the $17 \mathrm{GHz}$ and $34 \mathrm{GHz}$ NoRH flux densities are obtained with 2-min averaging, we also smooth the NoRP light curves over a 2-min scale. Figure 2 shows that after $\sim 6: 00$ UT the NoRH flux density of the impulsive component is close to 0 at $17 \mathrm{GHz}$.

Below $9.4 \mathrm{GHz}$, self-absorption is significant, and the gradual component can be subtracted from the $2 \mathrm{GHz}$ and $3.75 \mathrm{GHz}$ light curves by assuming that the spectrum of the gradual component does not change with time. We can multiply the $34 \mathrm{GHz}$ flux density by an absorption factor and subtract it from the total flux density. In practice, this factor is adjusted so that the flux density of the impulsive component is close to 0 after $\sim 6: 00 \mathrm{UT}$. At $2 \mathrm{GHz}$ and $3.75 \mathrm{GHz}$, we find a factor of 0.27 and 0.49 , respectively. Figure 2 shows that the impulsive components at different radio frequencies (solid lines) are also well correlated.

More information can be recovered from radio images at 17 and $34 \mathrm{GHz}$. Figure 3 shows the SDO/HMI magnetic field along the line of sight, which varies between $\sim 1500$ gauss and $\sim 1500$ gauss. It can be seen that active region 12403 has a complex magnetic field structure. Overplotted on these images are contours of radio images at 17 and $34 \mathrm{GHz}$ at four time intervals: in the pre-heating phase, at the peak of the impulsive emission, at the peak of gradual emission, and in the decay phase. To obtain these contours, NoRH radio images are integrated over a 2-min 

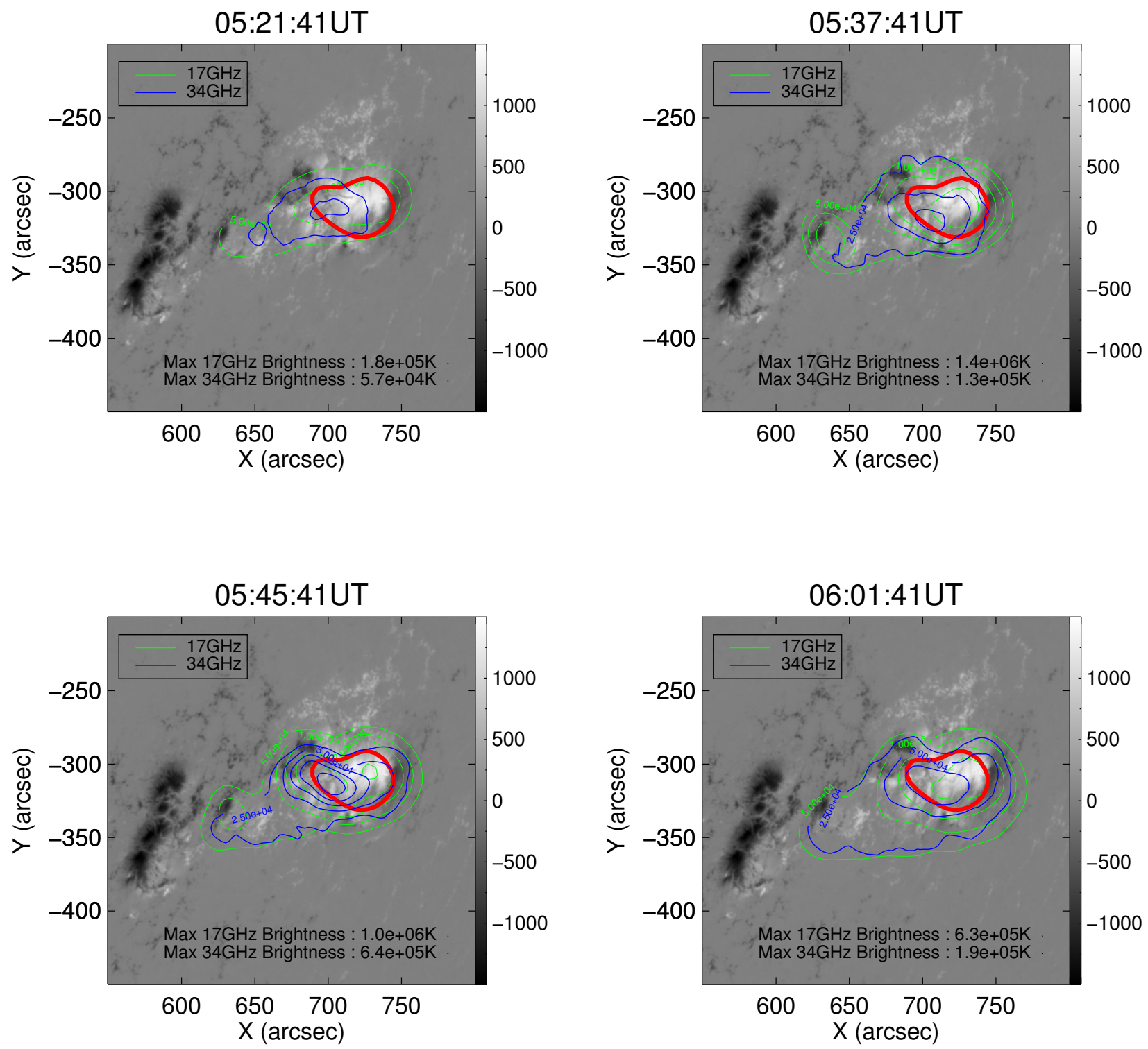

Fig. 3. NoRH images of the flare in the pre-heating phase (5:21:41 UT), at the peak of the impulsive (5:37:41 UT) and gradual (5:45:41 UT) emission, and in the gradual decay phase (6:01:41 UT). The background is the SDO/HMI images of the line-of-sight magnetic field ranging between about -1500 gauss and 1500 gauss. The red contour, the same as that in the inset in Fig. 2, marks the boundary of an emission region with the $17 \mathrm{GHz}$ brightness temperature greater than $4 \times 10^{5} \mathrm{~K}$ at the peak of the impulsive phase. The contours at $17 \mathrm{GHz}$ and $34 \mathrm{GHz}$ start at $5 \times 10^{4} \mathrm{~K}$ and $2.5 \times 10^{4} \mathrm{~K}$, respectively. The contours are separated from each other by a factor of 2 . The maximum brightnesses are indicated in the figures.

period to enhance the figure quality; the center times of these integration periods are indicated in the figures. We note that the pre-flare image of the active region is not subtracted from these radio images.

In the pre-heating phase, images at $17 \mathrm{GHz}$ and $34 \mathrm{GHz}$ are closely correlated and enhanced radio emission can be seen above active region 12403. At the peak of the impulsive phase, the $17 \mathrm{GHz}$ flux is dominated by a bright source in a direction with strong positive (i.e., pointing toward the observer) light-of-sight magnetic fields in the photosphere, which may be identified as the impulsive component. In addition to a dominant source to the west, there is a weak feature extended to the east in the $17 \mathrm{GHz}$ image. These two sources can also be seen in the $34 \mathrm{GHz}$ image, where the fainter one is aligned with the bright source at $17 \mathrm{GHz}$ and the other brighter one is aligned with the weak feature extended to the east. The peak of the gradual emission is dominated by the eastern source at both
$34 \mathrm{GHz}$ and $17 \mathrm{GHz}$, though emission from the western source is still visible. In the decay phase the $34 \mathrm{GHz}$ emission is still dominated by the eastern source, while the $17 \mathrm{GHz}$ emission is dominated by the western source. By comparing the peak brightness temperatures at the peak of the gradual emission and the decay phase (low panels in Fig. 3), we can see that the eastern source actually decays faster than the western source. The differences between the $17 \mathrm{GHz}$ and $34 \mathrm{GHz}$ images suggest different emission mechanisms, in agreement with differences in their light curves.

Since the impulsive component has a higher peak flux density and a shorter variation timescale at $17 \mathrm{GHz}$, we expect a higher brightness temperature of this emission component. The red contours in Fig. 3 mark an emission region with a brightness temperature greater than $4 \times 10^{5} \mathrm{~K}$ in the $17 \mathrm{GHz}$ image at the peak of impulsive emission. The red lines in Fig. 2 show the $17 \mathrm{GHz}$ (solid) and $34 \mathrm{GHz}$ (dot-dashed) flux density from such 
an emission region. Indeed, the impulsive component of $17 \mathrm{GHz}$ emission is dominated by this bright source. Although the overall flux densities at $17 \mathrm{GHz}$ and $34 \mathrm{GHz}$ are similar in the decay phase, radio images show that the impulsive component decays gradually and contributes to the total flux density at $17 \mathrm{GHz}$ in the late decay phase after 6:00 UT, which suggests that using the correlation of light curves alone to separate the impulsive and gradual emission components may not be applicable in the decay phase. Since we focus on the impulsive phase, these caveats do not affect our results and conclusions.

The main phase of this radio flare may therefore be attributed to three emission components: the impulsive component to the west dominates the impulsive phase, but decays very slowly at $17 \mathrm{GHz}$; the gradual component to the east dominates at the peak of the gradual emission and the emission at $34 \mathrm{GHz}$ (in the sense that it has the highest brightness) and decays quickly; and the gradually evolving extended component with relatively low surface brightness.

\section{Spectral analyses}

The gradual emission is mostly produced via the bremsstrahlung process with a flat spectrum in the radio band, which explains the good correlation of gradual emission at radio, EUV, and X-ray bands. Although the $17 \mathrm{GHz}$ and $34 \mathrm{GHz}$ images have some differences, in the pre-heating phase their brightnesses (which are proportional to the product of the brightness temperature and the square of emission frequency) are comparable, giving rise to comparable flux densities (Iwai \& Shibasaki 2013). During the main phase of the flare, the brightness of the eastern source at $34 \mathrm{GHz}$ is actually higher than that at $17 \mathrm{GHz}$, implying selfabsorption of the emission at $17 \mathrm{GHz}$.

However, these brightness temperatures are much lower than the temperature of plasma inferred from GOES observations; the gradual radio emission of the eastern source therefore should be dominated by a plasma with a temperature comparable to the brightness temperature of the $17 \mathrm{GHz}$ emission, which is consistent with flux density analyses in Sect. 2 (third and fifth panels of Fig. 1).

The impulsive radio and X-ray emissions are produced by energetic electrons via the gyro-synchrotron and bremsstrahlung processes, respectively (Dulk 1985; White et al. 2011). The eastern source is partially self-absorbed at $17 \mathrm{GHz}$, which means that the $17 \mathrm{GHz}$ flux density of the impulsive emission component derived in the previous section should be treated as a lower limit for gyro-synchrotron emission since the $34 \mathrm{GHz}$ flux density of the eastern source (which is subtracted from the $17 \mathrm{GHz}$ flux density) is slightly higher than that at $17 \mathrm{GHz}$. The total $17 \mathrm{GHz}$ flux density of the impulsive and gradual components together can be treated as an upper limit for the gyro-synchrotron emission. Moreover, since no impulsive emission is seen at $34 \mathrm{GHz}$, the flux density of $34 \mathrm{GHz}$ emission from the bright emission region may be treated as an upper limit for the gyro-synchrotron emission.

Therefore, the flux density of the impulsive emission component at $17 \mathrm{GHz}$ is always higher than that at $34 \mathrm{GHz}$ before 5:40 UT, consistent with a predominately gyro-synchrotron origin for impulsive $17 \mathrm{GHz}$ radio emission.

Radio emissions at $9.4 \mathrm{GHz}$ and lower frequencies show a prominent peak for the impulsive component and there is no evident flux increase associated with the peak of the gradual emission at 5:45 UT (Fig. 1). From the above analysis, the impulsive component should be dominated by gyro-synchrotron emission, while the gradual component has contributions from both

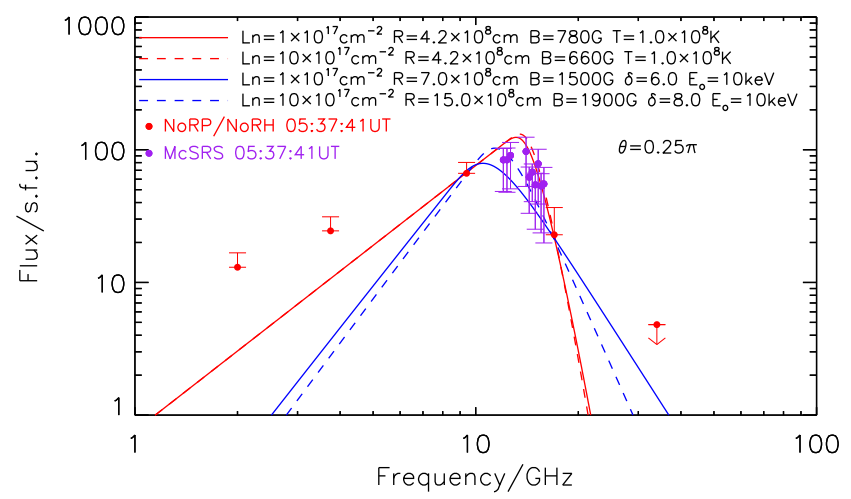

Fig. 4. Radio spectrum of the flare at the peak of impulsive emission 05:37:41 UT. The upper and lower bounds of the 2, 3.75, 9.4, and $17 \mathrm{GHz}$ flux densities are for the whole flare (dot-dashed) and the impulsive (solid) component in Fig. 2, respectively. The upper bound of the $34 \mathrm{GHz}$ data corresponds to the flux density from the bright region with the brightness temperature higher than $4 \times 10^{5} \mathrm{~K}$ at the peak of impulsive $17 \mathrm{GHz}$ emission (red dot-dashed line in Fig. 2). The purple data show observations with the Mengcheng Solar Radio Spectrometer (McSRS). The lines show model fits to the spectrum. See text for details.

the bremsstrahlung and the gyro-synchrotron process. For the bremsstrahlung emission, there should be strong self-absorption at these low frequencies because the emission is already selfabsorbed at $17 \mathrm{GHz}$. By comparing flux densities of the impulsive component at different radio frequencies, we can see that the gyro-synchrotron emission should be optically thin above $17 \mathrm{GHz}$ and optically thick below 9.4 GHz. The emission should peak near $10 \mathrm{GHz}$. To have a better constraint on the distribution of energetic electrons producing the gyro-synchrotron emission, we can treat the flux densities of the impulsive component as lower limits to the $2,3.75,9.4$, and $17 \mathrm{GHz}$ flux densities produced via the gyro-synchrotron process, and the total flux densities as upper limits.

Figure 4 shows the radio spectrum of the impulsive component at the peak of the impulsive emission. The $17 \mathrm{GHz}$ flux density of the impulsive emission is greater than 20 solar flux units (sfu). The corresponding $34 \mathrm{GHz}$ flux density of the bright emission region is about $5 \mathrm{sfu}$. Given the smooth evolution of the flux density at $34 \mathrm{GHz}$, this emission should be dominated by the bremsstrahlung process. Therefore, the flux density of 5 sfu should be considered as an upper limit to the impulsive gyrosynchrotron emission at $34 \mathrm{GHz}$. The spectral index of the gyrosynchrotron emission between $17 \mathrm{GHz}$ and $34 \mathrm{GHz}$ should be greater than 2 . If the emission is produced by electrons with a power-law distribution, the index $\delta$ of the electron distribution should be greater than $(2+1.22) / 0.9=3.6$, which would lead to a hard X-ray photon index greater than 4.1 and 2.1 for the thin and the thick target model, respectively (Brown 1971).

Figure 5 shows the X-ray spectral fits in the $10-100 \mathrm{keV}$ range. A two-thermal and a thermal plus power-law model both give comparable fits to the observed count spectra. Considering the poor count statistics at high energies in the pre-heating and decay phases, the temperature of the high-temperature component of the two-thermal model and the power-law index of the thermal plus power-law model are fixed at $4 \mathrm{keV}$ and 6.5 , respectively, except for the impulsive phase.

The power-law model has a photon index between 6.0 and 6.5 , which is consistent with a constant value of 6.5 considering the errors (Fig. 6). However, in the 10-100 keV range, the emission is dominated by the power-law component during 

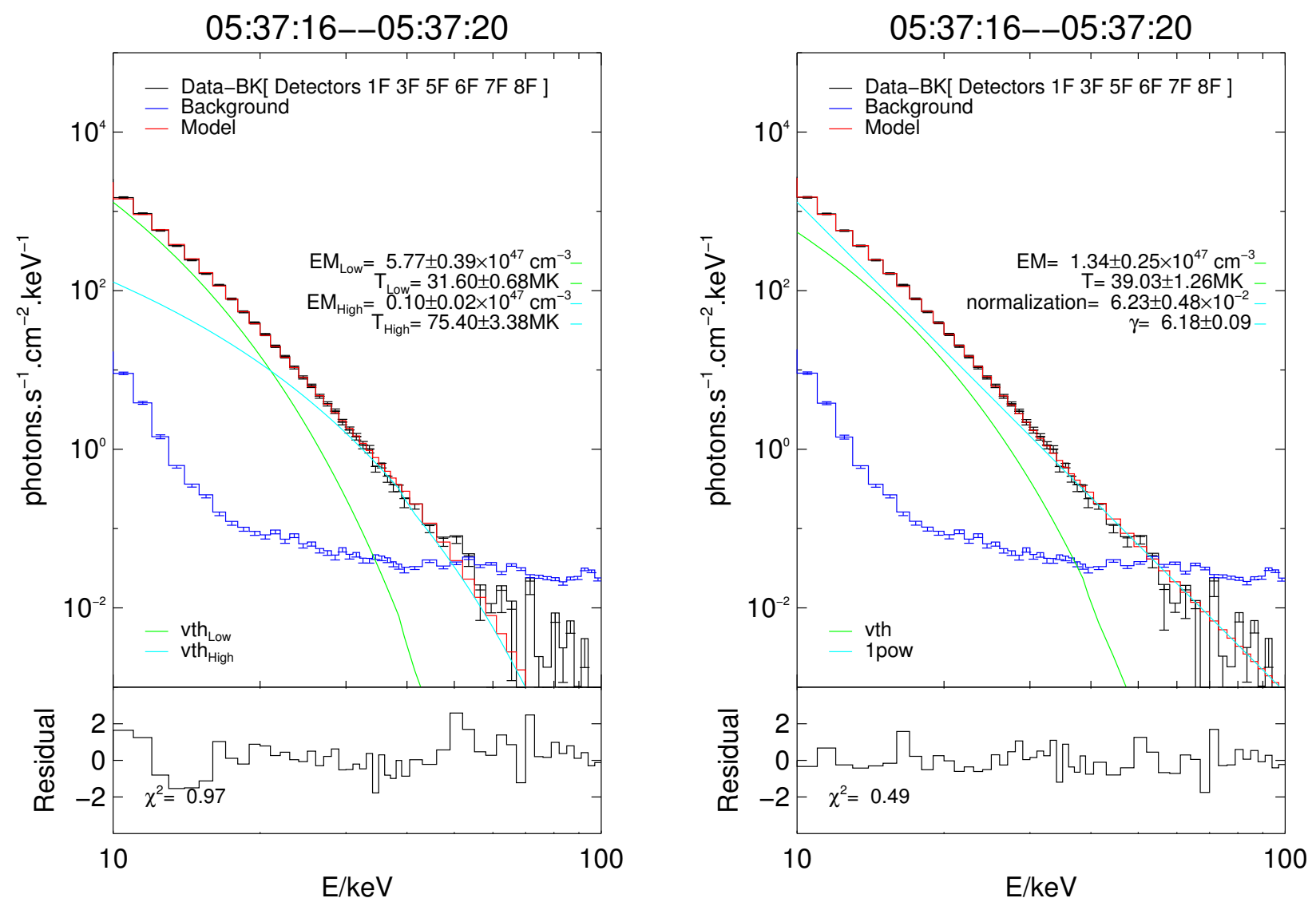

Fig. 5. RHESSI X-ray spectral fits in the $10-100 \mathrm{keV}$ range. The left and right panels correspond to a two-thermal model and a thermal plus power-law model, respectively, showing the spectral fits to the 05:37:16-05:37:20 interval with the corresponding model parameters indicated.

the impulsive phase (Fig. 5) and the impulsive flux variation is attributed to variation in the normalization of the power-law component. The temperature and emission measure of the thermal component show complex evolution during the impulsive phase. The hotter thermal component in the two-thermal model has a temperature up to $9 \mathrm{keV}$, which is about $T=100 \mathrm{MK}$, hot enough to produce strong gyro-synchrotron emission. The corresponding radio spectral index is about 8 , in agreement with radio flux density measurements. The model parameters vary smoothly with the impulsive flux increase attributed to an increase in the temperature of the hotter component ${ }^{1}$. The two-thermal model is therefore preferred to the thermal plus power-law model.

If we model the radio spectrum of the impulsive component with a uniform face-on cylinder with a radius $R$ and a height $L$, we can estimate the magnetic field (Dulk 1985). The corresponding results are shown in Fig. 4 assuming an effective angle of $45^{\circ}$ between the line of sight and the magnetic field. Since for a given temperature the thermal gyro-synchrotron emissivity is very sensitive to the magnetic field, a higher column density $L n$ is needed for a lower magnetic field to produce a nearly identical emission spectrum. From the emission measure of $\sim 10^{46} \mathrm{~cm}^{-3}$ for the hotter plasma at the peak of the impulsive emission, we get a density of $n \sim 10^{9} \mathrm{~cm}^{-3}$ for a volume of $\sim 10^{28} \mathrm{~cm}^{3}$; the corresponding column density is about $10^{18} \mathrm{~cm}^{-2}$. We therefore infer a magnetic field of $\sim 600$ gauss, which is compatible with SDO/HMI magnetic field measurements, indicating that the emission is produced not far from the photosphere. For an X-ray photon index

\footnotetext{
1 The change in attenuators indicated in the top panels of Fig. 6 causes a jump in model parameters, which is clearly an instrumental effect.
}

of 6.5, the emitting electrons have an index of 6 and 8 (with a low-energy cutoff $E_{0}=10 \mathrm{keV}$ ) for the thin and thick target model, respectively. These power-law models require a magnetic field stronger than 1500 gauss, which is close to the maximum of the photospheric value, which is not very reasonable. The radio flux density measurements (purple points in Fig. 4) taken with the Mengcheng Solar Radio Spectrometer also favor the thermal model. The simple uniform face-on cylinder model underestimates the radio flux density below $9.4 \mathrm{GHz}$, implying a more complex source structure. The power-law model deviates further from the observation than the thermal model in the low frequency range.

In summary, although a two-thermal and a thermal plus power-law model can both give reasonable fits to the 10-100 keV count spectra, the evolution of model parameters and radio spectral fit at the peak of the impulsive emission favor the two-thermal model.

\section{EUV and X-ray images and magnetic field extrapolation}

Due to the thermalization of the energy deposited at flare footpoints in the impulsive phase, thermal impulsive emission is expected at footpoints. Indeed, with early Yohkoh observations, Hudson et al. (1994) first reported impulsive soft X-ray emission from flare footpoints. The thermal nature of this emission is better established with late Hinode/Extreme Ultraviolet Imaging Spectrometer observations (Graham et al. 2013) and joint observations via SDO/AIA and RHESSI (Simões et al. 2015). However the temperature of thermal emission from 
Ping Zhang et al.: Impulsive radio and hard X-ray emission from an M-class flare
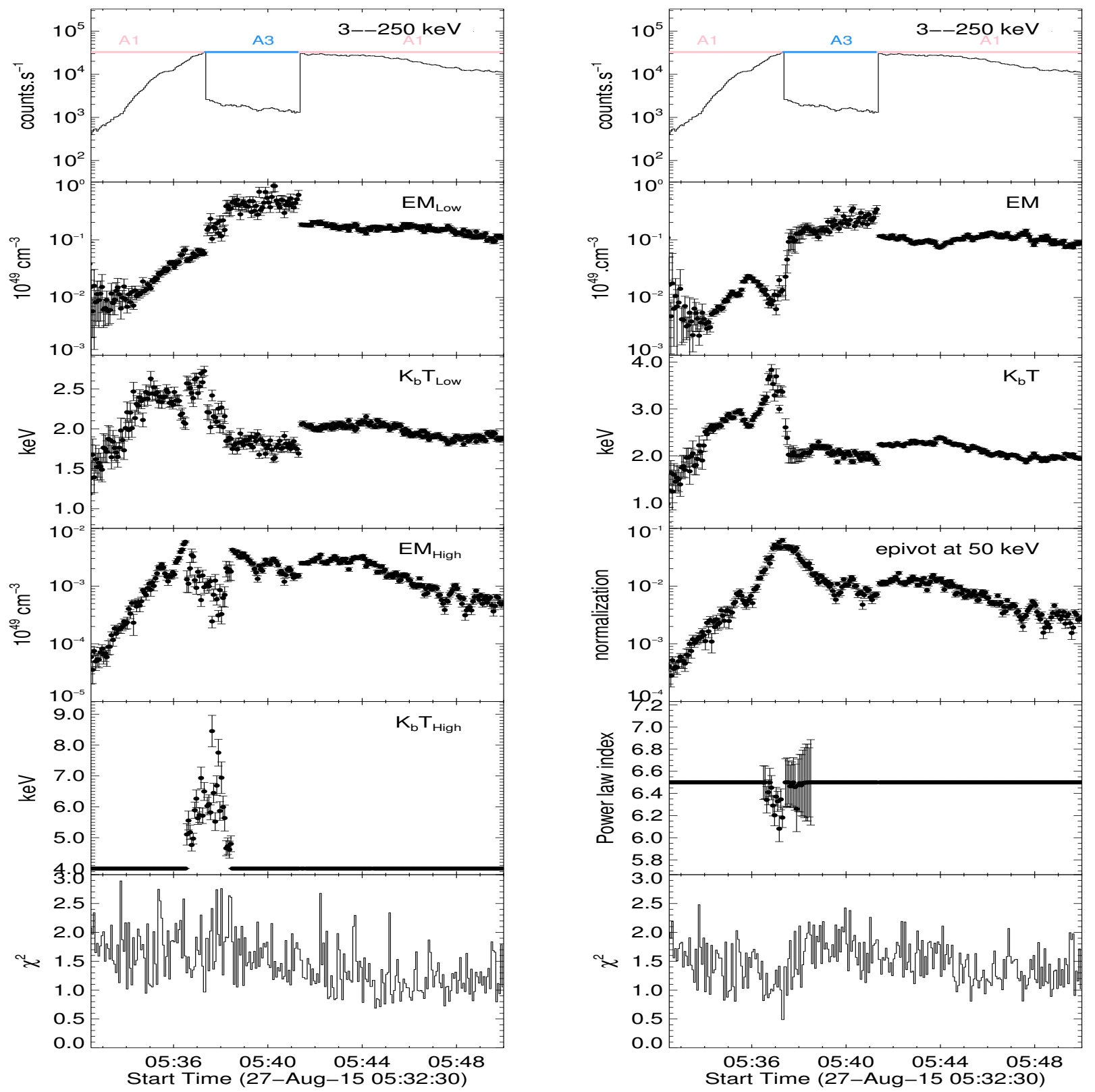

Fig. 6. Evolution of the parameters of a two-thermal (left) and a thermal plus power-law (right) model. In the pre-heating and decay phases, the temperature of the high-temperature component of the two-thermal model and the power-law index of the thermal plus power-law model are fixed at $4 \mathrm{keV}$ and 6.5, respectively, due to poor count statistics at high energies. The last panels show the evolution of the reduced $\chi^{2}$ of the spectral fits.

footpoints is usually less than $1 \mathrm{keV}$. If the impulsive emission of the flare studied here originates from a hot thermal plasma with a temperature higher than $4 \mathrm{keV}$, the emission should come primarily from the corona instead of the chromosphere.

Although the density estimate in the previous section supports a corona origin, imaging observations in the X-ray and EUV bands can give more direct constraints. Figure 7 shows the SDO/AIA multiwavelength images of the flare at the four periods shown in Fig. 3 with the corresponding $17 \mathrm{GHz}$ radio contours overplotted. The neutral line of the line-of-sight magnetic field is also indicated in the figure. The Hinode/XRT image at 05:18 UT is very similar to the AIA image at the pre-heating phase and is not shown here. In the pre-heating phase, the flare region already shows a complex magnetic field structure. The coronal magnetic field is highly twisted, and these twisted magnetic fields appear to relax to a more structured arcade of loops as the flare evolves. The brightest EUV source sits in the middle of the flare region. Although its western part is spatially coincident with a region with positive magnetic polarity, the hot flaring plasma is likely located in the corona, as is evident from the last AIA image for the decay phase. The footpoints of negative magnetic field are located along a ribbon very close to the magnetic neutral line, as is evident in the image of the peak of the gradual emission in Fig. 7. Most footpoints of positive magnetic field are also aligned along an arc that is a bit to the northeast of the impulsive component identified from radio observations, as is evident in the $304 \AA$ image of the peak of the impulsive emission. As the flare evolves, these footpoints do not appear to move significantly. However, different segments of the footpoints play the dominant role in different phases of the flare. At the peaks of the impulsive and the gradual emission, we also overplot contours 

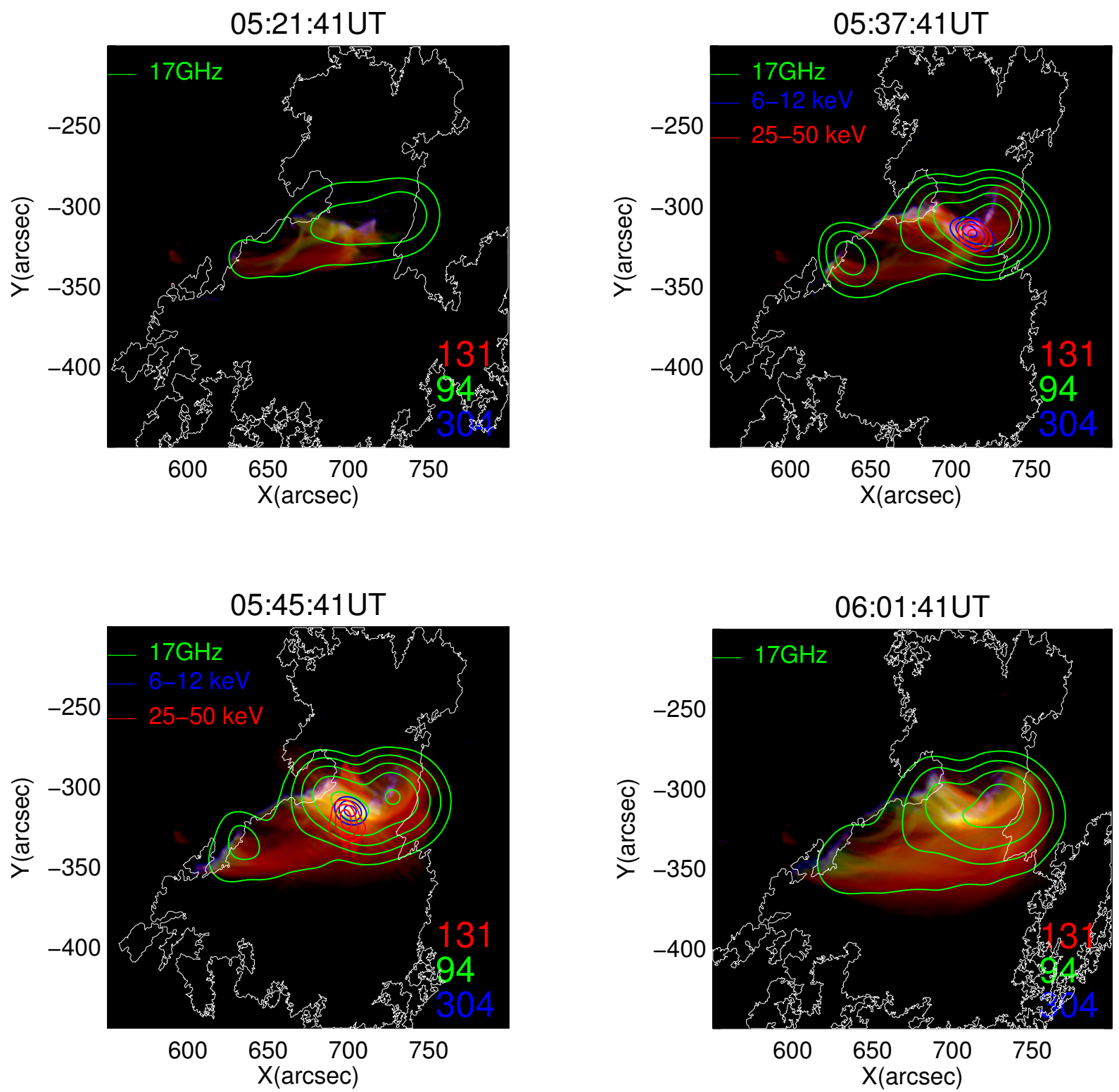

Fig. 7. SDO/AIA multiwavelength images for the four periods shown in Fig. 3. Contours of the corresponding $17 \mathrm{GHz}$ images are overplotted. To facilitate comparison between EUV and radio images, the AIA images are also averaged over a 2-min period. At the peak of the gradual emission, some of the AIA images saturate. The white line marks the neutral line of the line-of-sight magnetic field. Contours of RHESSI images at 6-12 keV and $25-50 \mathrm{keV}$ are also shown for the flux peaks of the impulsive and the gradual emission. The contour levels are $90 \%, 70 \%$, and $50 \%$ of the corresponding peak brightness. The front segment of detectors $4,5,6,7$, and 8 are used to construct the RHESSI image with the CLEAN algorithm with an integration time of $20 \mathrm{~s}$.

of RHESSI images at 6-12 keV and $25-50 \mathrm{keV}$. The 6-12 keV contours are always aligned with the $304 \AA$ emission which originates from the footpoints. The $25-50 \mathrm{keV}$ contours are always to the south of the low-energy $6-12 \mathrm{keV}$ contours which is consistent with a corona origin of the $25-50 \mathrm{keV}$ emission.

To further demonstrate the position of the radio and X-ray sources relative to the footpoints, Fig. 8 shows IRIS images at the peaks of the impulsive and the gradual emission with the corresponding radio and X-ray contours overplotted. The lowenergy 6-12 keV X-ray emission is always aligned with the footpoints.

At the peak of impulsive emission, the $25-50 \mathrm{keV}$ hard X-ray source is located between the impulsive and gradual emission components identified with radio observations and is slightly to the west of the IRIS and soft X-ray source. At the peak of gradual emission, it is slightly to the south of the gradual radio source and the bright EUV source. These X-ray images are consistent with a corona origin of the hard X-ray emission. The impulsive radio source is always slightly to the southwest of the footpoint ribbon implying a coronal origin.

Although the impulsive radio emission is closely correlated to the impulsive hard X-ray emission in the $25-50 \mathrm{keV}$ band, the hard X-ray image of the impulsive component is shifted to the east of the impulsive radio component. This can be readily explained with the emission mechanisms discussed above. The impulsive radio emission is produced by high-energy electrons via the gyro-synchrotron process, which is sensitive to the magnetic field strength; instead the impulsive X-ray emission is produced by high-energy electrons via the bremsstrahlung process, which is proportional to the density of the background plasma. Although the impulsive $17 \mathrm{GHz}$ emission is well aligned with the southern IRIS ribbon, the X-ray source structure and AIA 

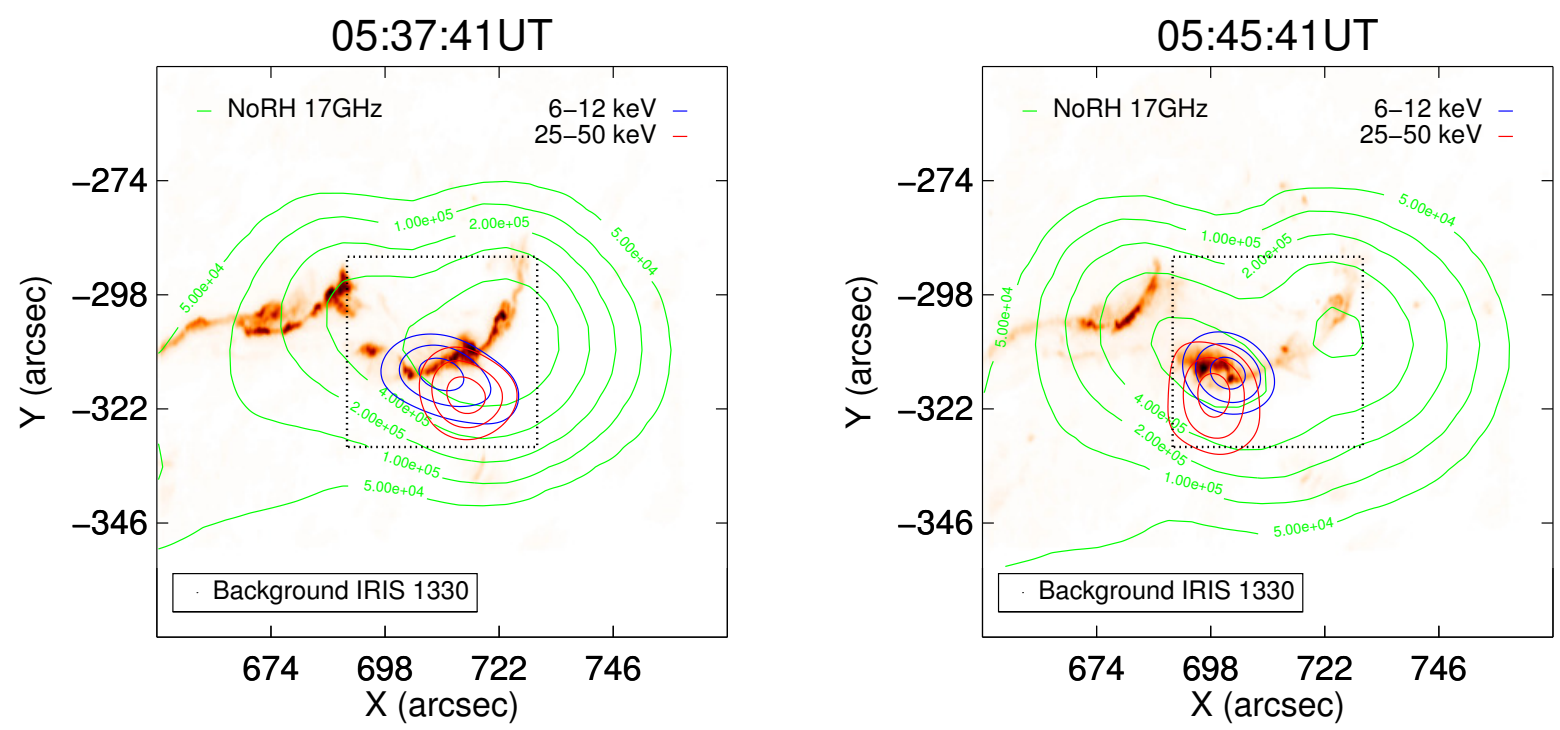

Fig. 8. IRIS images at the peaks of the impulsive (left) and the gradual emission (right). The overplotted $17 \mathrm{GHz}$ radio and X-ray contours are the same as those in Fig. 7.

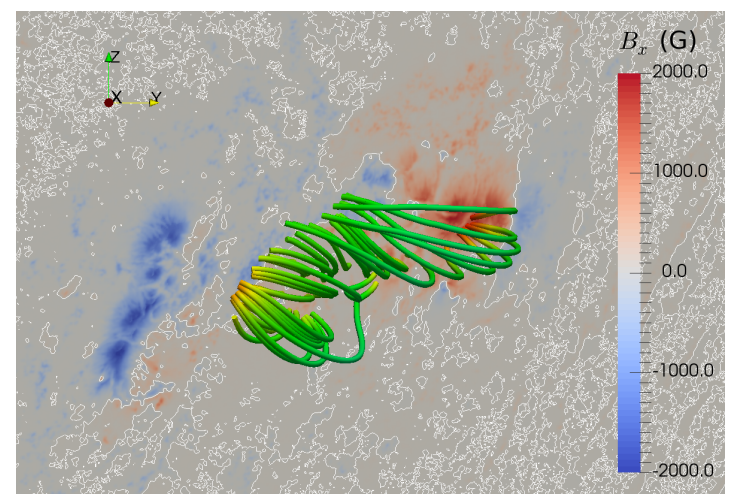

Fig. 9. Magnetic field lines obtained via a nonlinear force-free extrapolation model.

images in Fig. 6 favor a coronal origin of the impulsive radio source, which is also slightly to the southwest of emission centroid of the IRIS footpoint ribbon.

To further explore the structure of the magnetic field, we use the nonlinear cforce-free model described in Guo et al. $(2016 b, a)$ to extrapolate the coronal magnetic field with the vector magnetic field in the photosphere. Figure 9 shows the corresponding result for the pre-heating phase (5:24 UT). The magnetic field structure is consistent with a coronal interpretation of the main radio, EUV, and X-ray sources.

In summary, the $6-12 \mathrm{keV}$ soft $\mathrm{X}$-ray sources are aligned with the footpoints. The $25-50 \mathrm{keV}$ hard X-ray source is always to the south of the footpoints, suggesting a corona origin of the hard X-ray emission. If we align the $25-50 \mathrm{keV}$ emission with the footpoints expected for the thick target model, then the 6-12 keV emission will not have a counterpart in the EUV and IRIS images. The impulsive radio emission is always to the southwest of the footpoints, which is also consistent with a corona origin.

\section{Discussion and conclusions}

To understand the radiation and particle energization processes of solar flares, we analyze multiwavelength observations of an M-class flare that occurred on August 27, 2015. The radio light curves of this flare, as observed by the Nobeyama radio polarimeter and the Nobeyama radio heliograph, have two distinct components: an impulsive and a gradual component, which are closely correlated with the RHESSI hard X-ray light curves and the GOES soft X-ray SDO/EVE light curves, respectively. The absence of impulsive emission at $34 \mathrm{GHz}$ and hard X-ray emission above $50 \mathrm{keV}$ indicates that the emission is produced by energetic electrons with a very soft distribution. In combination with spectral analyses, imaging observations, and extrapolation of coronal magnetic field structure with a nonlinear force-free model, we conclude that the impulsive emission is produced by super-hot plasmas with a temperature of $\sim 100 \mathrm{MK}$ confined in coronal loops. Although a thermal plus power-law model can give acceptable fits to the X-ray spectra, the model is disfavored because of the dominance of the power-law component over the thermal component at low energies (which is not very common), the complex evolution of model parameters in the impulsive phase, and the radio spectral fit. The X-ray source structure disfavors a thick target model.

This temperature is a few times higher than that of the plasma probed with GOES soft X-ray flux measurements and may be produced if the particle energization process is dominated by betatron acceleration of contracting loops (Bogachev \& Somov 2007). The radio spectral fit to the impulsive radio emission at the flux peak reveals a magnetic field of $\sim 600$ gauss, which is also consistent with a contraction of a factor of a few in the transverse direction of magnetic loops. Such a small contraction factor will lead to a soft power-law distribution in a narrow energy range even if the Fermi process associated with the contraction of magnetic loops in the longitudinal direction dominates for a thermal injection. Such a distribution might not be distinguished from a super-hot thermal distribution. The complex structure of the coronal magnetic field in the pre-heating phase as revealed with the SDO/AIA image supports dominance of the energization by collapsing magnetic traps. A compression factor of a few of a pre-heated plasma in the corona will increase both the temperature and emission measure of this plasma by the same factor, which can readily reproduce the impulsive hard X-ray emission observed in the $25-50 \mathrm{keV}$ band. This super-hot plasma has an energy content of $\sim 10^{29}$ erg, which is several times lower than another hot component with a temperature a few 
times lower than this super-hot component. The fast decrease in the impulsive radio and hard X-ray flux can be attributed to cooling of the super-hot plasma by surrounding hot plasmas via thermal conduction. The more gradual decay of the impulsive emission component identified via radio observations may be associated with high-energy electrons with relatively large pitch angles so that they can be trapped in coronal loops for a long time. If the enhanced X-ray emission in the main phase of this flare is dominated by the contraction of flare loops, we expect weaker evaporation from the chromosphere footpoints. $\mathrm{X}$-ray images indicate that high-energy emission comes from a high altitude supporting such a scenario. More analyses of IRIS observations may give a quantitative constraint on the evaporation process.

The NoRH images show that the impulsive component is dominated by a compact region with a high brightness temperature $\left(\geq 4 \times 10^{5} \mathrm{~K}\right.$ at the peak of impulsive $17 \mathrm{GHz}$ emission). The gradual emission is mainly associated with an extended region with lower brightness temperatures. Radio images also reveal a bright gradual emission component to the east of the impulsive component, which has EUV, soft X-ray, and hard $\mathrm{X}$-ray counterparts. Although we attribute both the impulsive radio and impulsive hard X-ray emission to a hot plasma, these two sources are spatially displaced by more than $10^{\prime \prime}$. This displacement can be attributed to their different mechanisms of emission. While the X-ray emission is produced via the bremsstrahlung process, which is sensitive to the density of the background plasma, the radio emission is produced via the gyro-synchrotron process, which is sensitive to the magnetic field.

We show that the hot plasma responsible for soft X-ray emission observed by GOES underproduces the gradual component of radio emission via the bremsstrahlung process (free-free emission) by a factor of $\sim 3$, indicating that the gradual radio emission is dominated by warm plasmas that do not significantly contribute to soft X-ray emission. The SDO/EVE observations support this interpretation. The RHESSI spectral analysis has already revealed multiple thermal components. More detailed analyses of SDO/AIA observations can give a quantitative measurement of the differential emission measure, which can be used to derive contributions to the gradual radio emission from different thermal components (Tsap et al. 2016). The flare in general has a complex magnetic field structure. Although a simple extrapolation of the coronal magnetic field structure with a nonlinear force-free model confirms the main conclusion of this paper, more detailed analyses are needed to uncover the topological evolution of the large-scale magnetic field.

Acknowledgements. This work is partially supported by the NSFC grants U1738122, 1171101143, 11233008 and by the International Partnership Program of Chinese Academy of Sciences, Grant No. 114332KYSB20170008, by the Strategic Priority Research Program on Space Science, the Chinese Academy of Sciences, Grant No. XDA15320301.

\section{References}

Bogachev, S. A., \& Somov, B. V. 2005, Astron. Lett., 31, 537 Bogachev, S. A., \& Somov, B. V. 2007, Astron. Lett., 33, 54

Brown, J. C. 1971, Sol. Phys., 18, 489

Chamberlin, P. C., Milligan, R. O., \& Woods, T. N. 2012, Sol. Phys., 279, 23

Dulk, G. A. 1985, ARA\&A, 23, 169

Eradat Oskoui, S., Neukirch, T., \& Grady, K. J. 2014, A\&A, 563, A73

Feng, L., Wiegelmann, T., Su, Y., et al. 2013, ApJ, 765, 37

Garcia, H. A. 1994, Sol. Phys., 154, 275

Graham, D., Hannah, I., Fletcher, L., \& Milligan, R. 2013, ApJ, 767, 83

Guo, J., Liu, S., Fletcher, L., \& Kontar, E. P. 2011, ApJ, 728, 4

Guo, Y., Xia, C., \& Keppens, R. 2016a, ApJ, 828, 83

Guo, Y., Xia, C., Keppens, R., \& Valori, G. 2016b, ApJ, 828, 82

Hudson, H., Strong, K., Dennis, B., et al. 1994, ApJ, 422, L25

Iwai, K., \& Shibasaki, K. 2013, PASJ, 65, S14

Kim, S., Nakariakov, V. M., \& Shibasaki, K. 2012, ApJ, 756, L36

Liu, S., Li, Y., \& Fletcher, L. 2013, ApJ, 769, 135

Musset, S., Vilmer, N., \& Bommier, V. 2015, A\&A, 580, A106

Sharykin, I., Liu, S., \& Fletcher, L. 2014, ApJ, 793, 25

Simões, P. J., Graham, D. R., \& Fletcher, L. 2015, Sol. Phys., 290, 3573

Somov, B. V., \& Kosugi, T. 1997, ApJ, 485, 859

Somov, B. V., Kosugi, T., Oreshina, I. V., \& Lyubimov, G. P. 2005, Adv. Space Res., 35, 1712

Tsap, Y. T., Smirnova, V. V., Morgachev, A. S., et al. 2016, Adv. Space Res., 57, 1449

Wang, L., Zhang, P., Liu, S. M., et al. 2017, AcASn, 58, A4

White, S. M., Benz, A. O., Christe, S., et al. 2011, SSR, 159, 225 